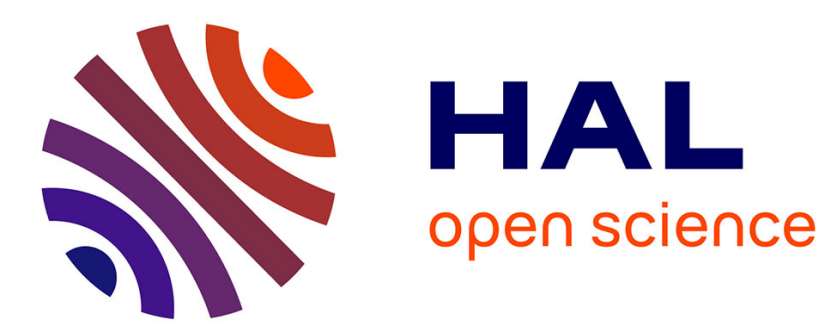

\title{
Metal-Insulator Transition and Local Structure of V2O3
}

\author{
A. Frenkel, E. Stern, F. Chudnovsky
}

\section{To cite this version:}

A. Frenkel, E. Stern, F. Chudnovsky. Metal-Insulator Transition and Local Structure of V2O3. Journal de Physique IV Proceedings, 1997, 7 (C2), pp.C3-1061-C3-1063. 10.1051/jp4:19972137 . jpa00255198

\section{HAL Id: jpa-00255198 https://hal.science/jpa-00255198}

Submitted on 1 Jan 1997

HAL is a multi-disciplinary open access archive for the deposit and dissemination of scientific research documents, whether they are published or not. The documents may come from teaching and research institutions in France or abroad, or from public or private research centers.
L'archive ouverte pluridisciplinaire HAL, est destinée au dépôt et à la diffusion de documents scientifiques de niveau recherche, publiés ou non, émanant des établissements d'enseignement et de recherche français ou étrangers, des laboratoires publics ou privés. 


\title{
Metal-Insulator Transition and Local Structure of $\mathrm{V}_{2} \mathrm{O}_{3}$
}

\author{
A.I. Frenkel, E.A. Stern and F.A. Chudnovsky* \\ Physics Department Box 351560, University of Washington, Seattle, WA 98195-1560, U.S.A. \\ * Ioffe Physical-Technical Institute, 19402 Saint Petersburg, Russia
}

\begin{abstract}
According to diffraction measurements of the average structure, $\mathrm{V}_{2} \mathrm{O}_{3}$ changes on heating from monoclinic to trigonal, accompanied by a $1.4 \%$ volume decrease, where some $\mathrm{V}-\mathrm{V}$ distances decrease by about $0.11 \AA$, favoring the Mott - Hubbard mechanism of the phase transition from insulator to metal. Our polarized XAFS measurements of the local structure of the single crystal $\mathrm{V}_{2} \mathrm{O}_{3}$ show the same decrease in volume but no change in local symmetry in the transition, indicating that the phase transition contains a significant order - disorder component, contrary to the purely displacive model based on diffraction results. XANES measurements found that the onset of the $1 s \rightarrow$ " $3 d$ " transition in the insulator shifts towards lower energies by about $1 \mathrm{eV}$ in the metal, consistent with the shielding of the core hole potential by conducting electrons.
\end{abstract}

\section{INTRODUCTION}

Vanadium sesquioxide $\left(\mathrm{V}_{2} \mathrm{O}_{3}\right)$ undergoes a phase transition at $155 \mathrm{~K}$ from an antiferromagnetic insulator to paramagnetic metal with the crystal structure changing from monoclinic (I2/a) to trigonal $(R \overline{3} C)$ with a $1.4 \%$ decrease in volume at elevated temperature. It is generally believed that the metal - insulator transition in $\mathrm{V}_{2} \mathrm{O}_{3}$ is driven by the Mott - Hubbard (MH) mechanism, consistent with an abrupt expansion of the vanadium atoms distances (by 0.11 $\AA$ in $\mathrm{V}_{2} \mathrm{O}_{3}$ ) as the temperature is lowered below $\mathrm{T}_{c}$. In this model, changes in $\mathrm{V}-\mathrm{V}$ distances regulate the balance between the positive intra-atomic Coulomb repulsion energy of the electrons $(U)$ and their bare band energy width $W$ by changing the overlap between $3 d \mathrm{~V}-\mathrm{V}$ orbitals, thus determining the bandgap [1]. It should be emphasized that since the MH mechanism depends on the distance between the first few neighboring atoms and the corresponding overlap of their wave functions, the measurements of the local structure may help to determine whether this or some other mechanism holds. The local structure can differ from the average periodic structure as measured by diffraction if disorder is present. The information of the local structure can be most reliably quantified using the x-ray absorption fine structure (XAFS) method [2], explaining the motivation for the present investigation. Indeed we do find a difference between the local and average structures as described below.

\section{EXPERIMENT AND RESULTS}

We performed single crystal measurements in order to enhance the sensitivity of XAFS to the local structure. Orienting the $c$ - axis parallel and perpendicular to the polarization $\vec{\epsilon}$ of the synchrotron radiation allows to better resolve the shortest V-V pair parallel to the $c$ - axis from other nearest neighbor V-V pairs which lie nearly in the basal plane.

The $\mathrm{V}_{2} \mathrm{O}_{3}$ crystals were grown in Ioffe Physical Technical Institute (St. Petersburg) by the Verneuil method. The powder for crystal growth was prepared by reducing high purity $(99.999 \%)$ ammonium metavanadate $\left(\mathrm{NH}_{4} \mathrm{VO}_{3}\right)$ in flowing hydrogen at $1000^{\circ} \mathrm{C}$ for $12 \mathrm{~h}$. The samples for measurements were cut from the crystals and after polishing and etching in hot $10 \% \mathrm{HNO}_{3}$ solution had the dimensions about $1.5 \times 2 \mathrm{~mm}^{2}$ and thickness $15 \mu \mathrm{m}$, (corresponding to the absorption edge step $\Delta \mu x \approx 2.5$ at $\mathrm{V} \mathrm{K}$-edge). To improve the stoichiometry, the samples were annealed at $1500^{\circ} \mathrm{C}$ at the partial pressure of oxygen of about $0.1 \mathrm{~m}$ Torr. The $a-c$ plane was parallel to the sample's surface.

XAFS measurements were performed at the National Synchrotron Light Source on beamline X11A at two temperatures below the phase transition for each orientation and two temperatures above. Cooling was performed using a Displex refrigeration system attached to a sealed copper cell containing the sample. Care was taken to prevent the crystal from fracturing on cooling due to the volume increase below the phase transition by attaching the sample onto a thin aluminized mylar film through the surface tension of a droplet of alcohol and attaching the mylar to the sample cell using vacuum grease, so that only the tip of a corner of the sample was touching the copper holder.

XAFS signals were analyzed by the UWXAFS software [3]. Theoretical scattering amplitudes and phases were generated using the FEFF6 code [4] for the monoclinic crystal structure model. Changes occurring in the local structure were parameterized in the fit process as corrections to interatomic distances. 

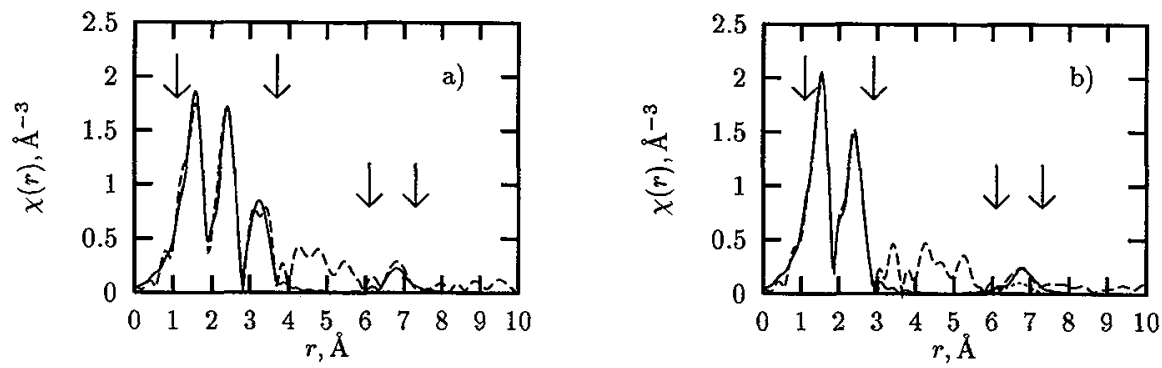

Figure 1: Fourier transforms of the $k^{2}$ - weighted XAFS spectra fitted with FEFF6 theory for monoclinic structure (solid) to the data (dash) at $130 \mathrm{~K}$ (a) and $200 \mathrm{~K}$ (b) for $\vec{\epsilon} \perp \vec{c}$ orientation. Fits with trigonal structure model to the $200 \mathrm{~K}$ data within the higher $r$ range are shown by dots. Fit ranges are indicated by arrows.

The monoclinic distortion of the trigonal structure [5] leads to the expansion of the one of the basal plane V-V distances by $0.11 \AA$. Fits to the data are shown in Fig. 1. The results of the fits (Fig. 2 a)) showed that the local structure of the samples remained monoclinic at all temperatures. The $V_{0}-V_{2}$ distance (between the pair of atoms lying nearly in the basal plane) decreased above the transition by $0.070 \pm 0.025 \AA$, but its absolute value remained $0.040 \pm 0.025 \AA$ longer than given by the trigonal structure of the diffraction measurement. Another characteristic distance (along the $c$-axis) between $V_{0}-V_{1}$ remained about $0.025 \pm 0.02 \AA$ longer than in the trigonal structure, consistent with the static displacement of $V_{0}$ and $V_{2}$ atoms in opposite directions in monoclinic structure.

To give further evidence of the lack of a purely displacive change in the local structure, we utilized the high sensitivity of XAFS signal to collinear arrangement of atoms [6]. The trigonal structure of $\mathrm{V}_{2} \mathrm{O}_{3}$ is characterized by a 6 - fold degeneracy of the collinearly arranged groups of three vanadium atoms, giving rise to the multiple scattering photoelectron paths with the same half path length $(7.38 \AA)$. In the monoclinic structure, the displacements of vanadium atoms from their positions in the trigonal lattice split this group into two groups of 4 - fold and 2 - fold degenerate collinearly arranged atoms with half path lengths of $7.47 \AA$ and $7.255 \AA$, respectively.
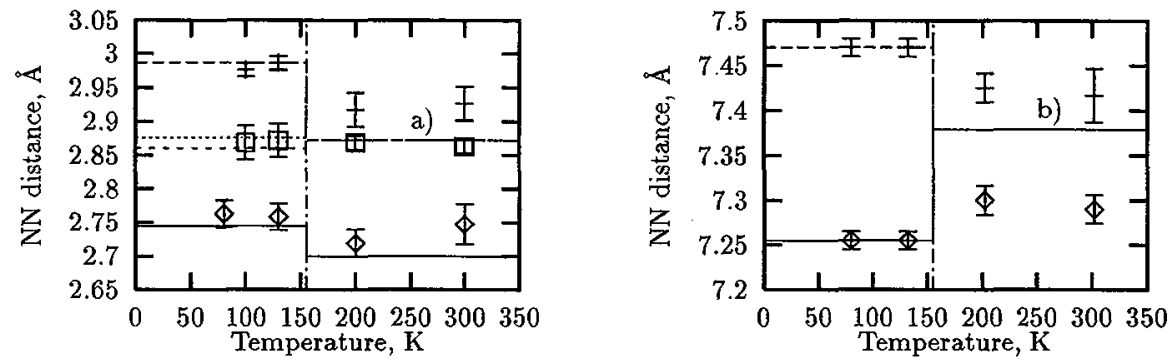

Figure 2: V-V distances as determined by x-ray diffraction (lines) and XAFS (symbols) at 5 temperatures below and above the phase transition (vertical line): a) - nearest neighbors, b) more distant neighbors.

The fit to the data within the range of $6.1-7.3 \AA$ is shown in Fig. 1 where the trigonal model was also used to fit the data in this range for comparison with the monoclinic model and gave much poorer fit quality. Figure $2 \mathrm{~b}$ ) shows that the two distances remain split above the phase transition. Monoclinic distortions, therefore, persist locally in the $\mathrm{V}_{2} \mathrm{O}_{3}$ above the phase transition temperature, though abruptly decreasing by $65 \pm 20 \%$ at the phase transition. The experimentally observed $1.4 \%$ decrease in the $\mathrm{V}_{2} \mathrm{O}_{3}$ volume, previously believed to be caused by the change in the unit cell dimensions between monoclinic and trigonal cells, can be explained mainly by the $\mathrm{V}_{0}-\mathrm{V}_{2}$ distances shortening in the monoclinic unit cell. Our estimates, using the change in $\mathrm{V}_{0}-\mathrm{V}_{2}$ distances obtained with XAFS, give $1.6 \pm 0.3 \%$ decrease in the volume of the monoclinic unit cell.

The average trigonal structure, as ascertained by diffraction, therefore, is a result of averaging over unoriented monoclinic regions, which are small enough (and may dynamically change orientation), so that they do not individually produce sharp monoclinic diffraction peaks. Our XAFS results can only ascertain a lower limit to the region of $7.3 \AA$ and give no information on the dynamics (the XAFS measurements are so fast $\left(\approx 10^{-15} \mathrm{sec}\right)$ that no motion of atoms can occur). While the monoclinic region size is big enough to be detected by diffraction at low temperature (where three-fold twinned domains of the monoclinic cell [5] occur) it changes abruptly above the transition.

Since the structure remains locally monoclinic, an analysis of XANES becomes especially important to understand the changes in electronic structure during the metal - insulator transition. The edge region is shown in Fig. 3 for all temperatures and both orientations. The data were aligned in absolute energy and normalized by an edge step. 
The pre-edge feature starting at about $5465 \mathrm{eV}$ corresponds to the $1 s \rightarrow$ " $3 d$ " dipole-forbidden transition which becomes allowed as a result of the hybridization of the $\mathrm{V} 3 d$ orbitals with the $\mathrm{O} 2 p$ orbitals [7]. The onset of the transition in $\vec{\epsilon} \perp \vec{c}$ orientation shifts towards lower energies in metallic phase relative to the insulating one by about $1 \mathrm{eV}$ (Fig. $3 \mathrm{~b})$ ), in an overall agreement with the result of Bianconi and Natoli [8]. This shift cannot be explained by the known value of the insulating gap of $\approx 0.1 \mathrm{eV}$ and the fact that no drastic changes were observed in the $3 \mathrm{~d}-$ band structures of $\mathrm{V}_{2} \mathrm{O}_{3}$ below and above the metal - insulator transition [9]. Instead, it most likely arises from the shielding of the potential of the core hole by conducting electrons in metallic phase and, therefore, reducing the energy of the $1 s \rightarrow$ " $3 d$ " transition.

The corresponding XANES region in the $\vec{\epsilon} \| \vec{c}$ orientation data (Fig. 3 a)) has no significant change between the insulating and metallic states. As discussed by Shin et al. [9], it is consistent with the extension of $e_{\pi}$ orbitals (which mainly govern the electronic properties in $\mathrm{V}_{2} \mathrm{O}_{3}$ ) perpendicular to the $\vec{c}$-axis.
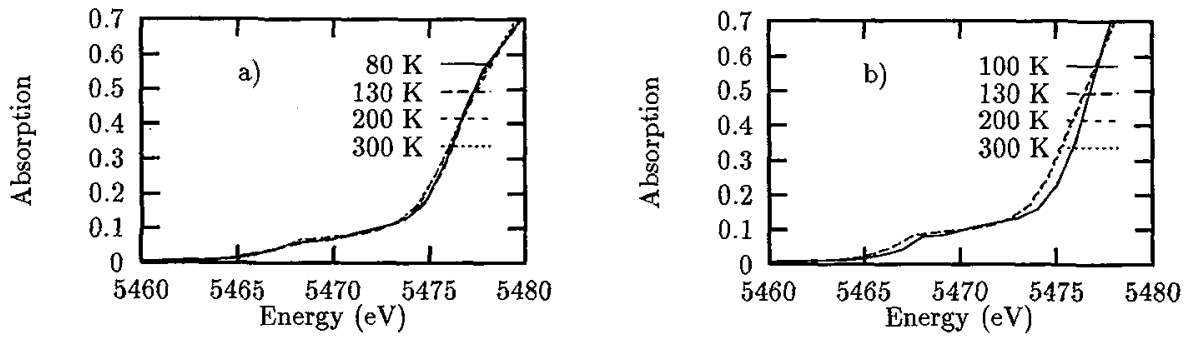

Figure 3: Near edge structure of the absorption coefficient below and above the phase transition for a) $\vec{\epsilon} \| \vec{c}$ and b) $\vec{\epsilon} \perp \vec{c}$.

\section{CONCLUSIONS}

In conclusion, our results show that the structural phase transition occurring in pure $\mathrm{V}_{2} \mathrm{O}_{3}$ is not the purely displacive transition with concomitant change of symmetry suggested by diffraction results. Rather, we obtained that the transition has both order - disorder and displacive character. The structure consists of locally monoclinic regions (static, or dynamically changing their orientation) which decrease their correlation lengths from infinite to between 7 - $40 \AA$ (where the monoclinic diffraction lines remain significantly broadened) above the metal - insulator transition, which leads to an average trigonal symmetry. The displacive character manifests itself above the transition by a decrease of monoclinic distortions within the domains by approximately $65 \%$.

XANES region in the metallic state of the $\vec{\epsilon} \perp \vec{c}$ orientation of $\mathrm{V}_{2} \mathrm{O}_{3}$ showed a $1 \mathrm{eV}$ shift of the $1 s \rightarrow$ " $3 d$ " transition towards lower energies, while the $\vec{\epsilon} \| \vec{c}$ orientation did not show any visible shift. This shift is consistent with shielding of the core hole potential by conducting electrons in the metallic state in the basal plane.

The wealth of information we obtained from these measurements should narrow the possible theoretical explanations. The role of disorder and the lack of local symmetry change in the transition to the metallic and paramagnetic states needs to be accounted for in any theory. Obtaining a better understanding of these materials may also contribute to the understanding of other classes of materials containing correlated electrons such as strongly correlated metals and high-temperature superconductors.

\section{Acknowledgments}

This work was supported by DOE Grant No. DE-FG06-90ER45425. Beamline X11A at NSLS is supported by DOE Grant No. DE-FG05-89ER45384.

\section{References}

[1] N. F. Mott, Metal - Insulator Transition, 2nd ed. (Taylor \& Francis, London, 1990) p. 176.

[2] E. A. Stern and S. M. Heald, in Handbook on Synchrotron Radiation, edited by E. E. Koch (North-Holland, New York, 1983), Vol. 1.

[3] E. A. Stern et al, Physica B 208 \& 209, 117 (1995); The UWXAFS software is licensed through the University of Washington. Further details can be obtained from E. A. Stern.

[4] S. I. Zabinsky et al., Phys. Rev. B 52, 2995 (1995).

[5] P. D. Dernier and M. Marezio, Phys. Rev. B 2, 3771 (1970).

[6] P. A. Lee and J. B. Pendry, Phys. Rev. B 11, 2795 (1975).

[7] J. Wong et al., Phys. Rev. B 30, 5596 (1984).

[8] A. Bianconi and C. R. Natoli, Sol. St. Comm. 27, 1177 (1978).

[9] S. Shin et al, Phys. Rev. B 41, 4993 (1990). 